\title{
Erratum to: Outcomes of trastuzumab therapy in HER2-positive early breast cancer patients
}

\author{
Hiroyasu Yamshiro $\cdot$ Hiroji Iwata $\cdot$ Norikazu Masuda $\cdot$ Naohito Yamamoto $\cdot$ Reiki Nishimura \\ Shoichiro Ohtani $\cdot$ Nobuaki Sato $\cdot$ Masato Takahashi $\cdot$ Takako Kamio $\cdot$ Kosuke Yamazaki \\ Tsuyoshi Saito $\cdot$ Makoto Kato $\cdot$ Tecchuu Lee $\cdot$ Shinji Ohno $\cdot$ Katsumasa Kuroi $\cdot$ \\ Toshimi Takano $\cdot$ Masahiro Takada $\cdot$ Shinji Yasuno $\cdot$ Satoshi Morita $\cdot$ Masakazu Toi
}

Published online: 14 March 2015

(C) Japan Society of Clinical Oncology 2015

\section{Erratum to: Int J Clin Oncol \\ DOI 10.1007/s10147-015-0785-8}

Unfortunately, the name of the 7th author was incorrectly published in the original article. The correct author name should read as Nobuaki Sato.

The online version of the original article can be found under doi:10.1007/s10147-015-0785-8.

H. Yamshiro $(\triangle)$

Department of Breast Surgery, Tenri Hospital, 200 Mishima-cho,

Tenri, Nara 632-8552, Japan

e-mail: yamashiro.bcs@gmail.com

\section{H. Iwata}

Department of Breast Oncology, Aichi Cancer Center Hospital,

1-1 Kanokoden, Chikusa-ku, Nagoya, Aichi 464-8681, Japan

e-mail: hiwata@aichi-cc.jp

\section{N. Masuda}

Surgery, Breast Oncology, NHO Osaka National Hospital,

2-1-14 Hohenzaka, Chuo-ku, Osaka 540-0006, Japan

e-mail:nmasuda@alpha.ocn.ne.jp

N. Yamamoto

Division of Breast Surgery, Chiba Cancer Center, 666-2 Nitona-cho, Chuo-ku, Chiba 260-8717, Japan

e-mail: nyamamot@chiba-cc.jp

\section{R. Nishimura}

Department of Breast and Endocrine Surgery, Kumamoto City

Hospital, 1-1-60 Kotoh, Higashi-ku, Kumamoto City,

Kumamoto 862-8505, Japan

e-mail: nishimura.reiki@cityhosp-kumamoto.jp
S. Ohtani

Breast Surgery, Hiroshima City Hospital, 7-33 Motomachi, Naka-ku, Hiroshima-sh, Hiroshima 730-8518, Japan e-mail: sho_ohtani@hotmail.com

N. Sato

Department of Breast Oncology, Niigata Cancer Center Hospital, 2-15-3 Kawagishi Town, Chuo-ku, Niigata 951-8566, Japan e-mail: nobus@niigata-cc.jp

M. Takahashi

Department of Breast Surgery, NHO Hokkaido Cancer Center, 4-2 Kikusui, Shiroishi-ku, Sapporo, Hokkaido 090-0011, Japan e-mail: masatotk@sap-cc.go.jp

\section{T. Kamio}

Department of Surgery II, Tokyo Women's Medical University, 8-1 Kawadacho, Shinjuku-ku, Tokyo 162-8666, Japan e-mail: ako@surg2.twmu.ac.jp

\section{K. Yamazaki}

Japanese Red Cross Hokkaido College of Nursing, 664-1 Akebono-cho, Kitami, Hokkaido 090-0011, Japan e-mail: kosyama@rchokkaido-cn.ac.jp 
T. Saito

Department of Breast Surgery, Saitama Red Cross Hospital, 8-3-33 Kamiochiai, Chuo-ku, Saitama-shi, Saitama 338-8553, Japan

e-mail: tsaito@jcom.home.ne.jp

\section{Kato}

Kato Breast Surgery Clinic, 8-12 Nishiojicho, Kusatsu, Shiga 525-0037, Japan

e-mail: k-chappy@symphony.plala.or.jp

T. Lee

Department of Breast Surgery, Japanese Red Cross Kyoto Daiichi Hospital, 15-749 Honmachi, Higashiyama-ku, Kyoto 605-0981, Japan

e-mail: chol-joo-lee@kyoto1-jrc.org

\section{S. Ohno}

Clinical Research Institute, NHO Kyusyu Cancer Center, 3-1-1 Notame, Minami-ku, Fukuoka 811-1395, Japan

e-mail:sohno@nk-cc.go.jp

\section{K. Kuroi}

Department of Breast Surgery, Tokyo Metropolitan Cancer and Infectious Diseases Center Komagome Hospital, 3-18-22 Honkomagome, Bunkyo-ku, Tokyo 113-8677, Japan e-mail: kurochan@dd.iij4u.or.jp

T. Takano

Department of Medical Oncology, Toranomon Hospital, 2-2-2 Toranomon, Minato-ku, Tokyo 105-8470, Japan

e-mail: takano@toranomon.gr.jp
M. Takada

Department of Surgery (Breast Surgery), Kyoto University Hospital, 54 Kawaharacho, Shogoin, Sakyo-ku,

Kyoto 606-8507, Japan

e-mail: masahiro@kuhp.kyoto-u.ac.jp

\section{S. Yasuno}

Department of EBM Research, Institute for Advancement of Clinical and Translational Science, Kyoto University Hospital, 54 Kawaharacho, Shogoin, Sakyo-ku, Kyoto 606-8507, Japan e-mail: syasuno@kuhp.kyoto-u.ac.jp

\section{S. Morita}

Department of Biomedical Statistics and Bioinformatics, Kyoto University Graduate School of Medicine,

54 Kawaharacho, Shogoin, Sakyo-ku, Kyoto 606-8507, Japan e-mail: smorita@kuhp.kyoto-u.ac.jp

M. Toi

Department of Surgery (Breast Surgery), Graduate School of Medicine, Kyoto University, 54 Kawaharacho, Shogoin, Sakyo-ku, Kyoto 606-8507, Japan e-mail: toi@kuhp.kyoto-u.ac.jp 